\title{
Essais
}

Revue interdisciplinaire d'Humanités

$8 \mid 2016$

Erreur et création

\section{«Les plus belles sont vicieuses »: erreur et fausseté dans La Manière de bien penser dans les ouvrages d'esprit du Père Bouhours (1687)}

\section{Raphaëlle Longuet}

\section{OpenEdition}

Journals

Édition électronique

URL : http://journals.openedition.org/essais/5197

DOI : 10.4000/essais.5197

ISSN : 2276-0970

Éditeur

École doctorale Montaigne Humanités

\section{Édition imprimée}

Date de publication : 15 mars 2016

Pagination : 108-123

ISBN : 978-2-9544269-7-6

ISSN : $2417-4211$

Référence électronique

Raphaëlle Longuet, « « Les plus belles sont vicieuses » : erreur et fausseté dans La Manière de bien penser dans les ouvrages d'esprit du Père Bouhours (1687) », Essais [En ligne], 8 | 2016, mis en ligne le 28 octobre 2020, consulté le 01 novembre 2020. URL : http://journals.openedition.org/essais/5197 ; DOI : https://doi.org/10.4000/essais.5197 


\section{« Les plus belles sont vicieuses »: erreur et fausseté dans La Manière de bien penser dans les ouvrages d'esprit du Père Bouhours (1687)}

\section{Raphaëlle Longuet}

En conclusion de son essai critique sur La Littérature de l'âge baroque en France $^{1}$, au moment de distinguer baroque et classicisme et pour mieux illustrer la complexité des rapports entre ces deux sensibilités qui se chevauchent à la fin du Grand Siècle, Jean Rousset choisit de se référer à un ouvrage méconnu des suffisants lecteurs contemporains : la Manière de bien penser dans les ouvrages d'esprit, du Père Bouhours². Ce dernier y aborde les principaux défauts du style à travers quatre dialogues entre deux amis, qui incarnent aux yeux du critique le Classicisme et le Baroque. Toutefois, la rivalité amicale qui caractérise habituellement leurs rapports se solde ici, une fois n'est pas coutume, par une victoire du premier sur le second, traité comme une " erreur " qu'il s'agirait de corriger.

Lorsqu'en 1687, le Père Bouhours publie la Manière de bien penser dans les ouvrages d'esprit, il a déjà sa place dans la vie littéraire de son temps : celle d'un "Jésuite homme de lettres " ${ }^{3}$ qui s'est illustré dans des genres et sujets variés, allant de la biographie de saint aux remarques grammaticales. Il est ainsi l'auteur d'une anthologie intitulée Recueil de diverses pièces sur les questions $d u$ temps (1668), d'une Vie de Saint Ignace (1679), de Doutes sur la langue française (1674), qui seront suivis de plusieurs volumes de Remarques nouvelles, ainsi que de divers recueils de Pensées et Sentiments chrétiens; mais ce sont avant tout ses Entretiens d'Ariste et d'Eugène, parus en 1671, qui lui acquièrent la notoriété

1 Jean Rousset, La Littérature de l'Âge baroque en France, Corti, 1953, p. 244-245.

2 Dominique Bouhours, La Manière de bien penser dans les ouvrages d'esprit, p. 12. Réédité en fac-similé par Suzanne Guellouz (Toulouse-Le Mirail, 1988), l'ouvrage sera cité tout au long du présent article dans son édition originale imprimée à Paris chez la Veuve Mabre-Cramoisy, rue St Jacques, 1687 (3722 BNF). L'orthographe a été modernisée, dans la continuité de la démarche de Bernard Beugnot et Gilles Declerq dans leur édition critique des Entretiens d'Ariste et d'Eugène (Paris, Champion, 2003).

3 L'expression est empruntée à Gérard Doncieux, auteur de la principale monographie consacrée au Père Bouhours, parue en 1886 (rééd. Genève, Slatkine reprints, 1970). 
dont il jouira jusqu'au XIX ${ }^{\mathrm{e}}$ siècle (comme en attestent les douze rééditions). "Microcosme du classicisme » selon Bernard Beugnot ${ }^{4}$, l'ouvrage rassemble en effet des sujets de prédilection de l'époque (la langue française, le je-ne-saisquoi, le bel esprit...) en associant érudition, variété et naturel grâce à la fiction d'un entretien entre deux honnêtes gens, qui devisent du bel esprit tout en l'illustrant. Ce principe est repris seize ans plus tard dans la Manière de bien penser dans les ouvrages d'esprit. L'auteur met à nouveau en scène deux amis devisant avec une érudition gardée du pédantisme par la liberté de ton des interlocuteurs, et le rejet en marge des passages en latin, " qui auraient embarrassé le discours, si on les y avait mêlés ». Mais le sujet en est cette fois plus uni, et le ton plus critique : si les personnages multiplient les citations, c'est à dessein de corriger les erreurs qui fourmillent dans les " ouvrages d'esprit".

L'originalité de la Manière de bien penser ne vient cependant pas tant de sa forme, qui illustre plutôt une tendance générale à faire participer les honnêtes gens de la cour aux débats poétiques. Plus surprenant est l'objet que se donne son auteur : alors que les questions dramatiques sont au cour des préoccupations de la critique, il n'est ici question que des " ouvrages d'esprit ", définis en préface comme « les histoires, les poèmes, les pièces d'éloquence, comme les harangues, les panégyriques, les oraisons funèbres, enfin tout ce qui s'écrit avec soin, et où il faut une certaine justesse qui va plus encore aux choses qu'aux paroles ». Cette préoccupation du juste rapport entre la langue et son objet se retrouve dès le premier dialogue, puisque la première exigence en matière de "bien penser » ne relève pas à proprement parler de ce qu'aujourd'hui l'on appellerait le style, mais plutôt, au premier abord, du fond :

Car enfin pour vous dire un peu par ordre ce que je pense là-dessus ; la vérité est la première qualité, \& comme le fondement des pensées : les plus belles sont vicieuses; ou plutôt celles qui passent pour belles, \& qui semblent l'être, ne le sont pas en effet, si ce fonds leur manque. ${ }^{5}$

Tout l'enjeu du premier dialogue va donc être de faire de la "fausseté " une préoccupation à part entière de la poétique, en la soulignant comme la première des erreurs. Ce critère du rapport de la création à la vérité confère à la critique une démarche objective qui l'inscrit dans une certaine modernité de la réflexion sur la langue.

Dès le portrait des deux amis, cependant, il apparait que l'entreprise de l'ouvrage consiste moins en un examen impartial des pensées qu'en l'opposition de deux esthétiques - Baroque, Classique - entre lesquelles l'auteur semble avoir déjà fait son choix. "Le caractère de leur esprit est bien différent ", nous dit-il : Eudoxe a " le goût très bon ", et son exigence est telle que " rien ne lui plait

4 Préface à l'édition critique des Entretiens d'Ariste et d'Eugène par Bernard Beugnot et Gilles Declerq, op. cit.

5 Dominique Bouhours, La Manière de bien penser..., op. cit., p. 12. 
dans les ouvrages ingénieux qui ne soit raisonnable et naturel "; ses auteurs de prédilection sont « les Anciens, surtout les Auteurs du siècle d'Auguste, qui selon lui est le siècle du bon sens". Philanthe, en revanche, aime " tout ce qui est fleuri, tout ce qui brille, le charme "; il se plait aux "Modernes " : "les Grecs et les Romains ne valent pas à son gré les Espagnols et les Italiens » Quand l'un cite Cicéron, Virgile, Voiture, l'autre répond Sénèque, Le Tasse, Balzac. Le choix onomastique cristallise cette opposition : Eudoxe suit la règle, et Philanthe son goût. Cependant, à la fin de l'ouvrage, Philanthe avoue de bon cœur s'être finalement rangé au goût de son ami : celui-ci " se réjouit " de le voir quitter " enfin [...] [ses] fausses idées ", et lui prodigue les derniers conseils qui l'empêcheront de " retomber dans [ses] anciennes erreurs " ${ }^{7}$.

Il est remarquable que ce dernier terme n'apparaisse quasiment que dans le dernier des dialogues, et comme en conclusion. Tout au long de l'ouvrage, l'auteur lui préfère les termes de "faute ", "pensée vicieuse ", "fausse pensée ", auxquels il oppose tout ce qui est " raisonnable » et pensé " correctement ». Un détour par les dictionnaires de l'époque permet de constater que le sémantisme actuel de l'erreur se partage alors entre deux substantifs. La " faute " exprime "l'action de la personne qui manque de quelque façon et en quelque sorte de chose que ce soit ". C'est sous ce terme que la langue classique entend toute erreur relative au langage : les « fautes contre la grammaire " côtoient ainsi les «fautes de jugement » des poètes dans les exemples donnés par Richelet ${ }^{8}$. Cette acception se trouve développée plus loin : "sans faute " signifie "sans qu'il y ait aucune faute, et selon toutes les règles. " La "fausseté " est ainsi la faute qui consiste à travestir la vérité : on trouve même chez Furetière l'entrée " faux brillant ", qui fait référence à " une pensée subtile qu’on a mise dans quelque ouvrage qui surprend agréablement l'esprit, et qui n'a point de solidité ». L' « erreur " connote davantage l' " hérésie ", la " méprise », la " folie »; c'est ce sens moral qui domine dans les exemples donnés par Furetière, pour qui "l'erreur est une méprise de l'entendement, qui donne son consentement à ce qui n'est pas véritable ${ }^{9}$. Le dialogue du Père Bouhours reflète cette ambivalence : Eudoxe corrige Philanthe comme seul un ami véritable peut oser le faire, sans flatterie, et avec un souci particulier qui laisse penser que les enjeux de leurs entretiens ne sont peut-être pas seulement esthétiques, mais aussi, indissociablement, éthiques.

\section{$6 \quad$ Ibid., p. 3.}

7 Ibid., p. 393.

8 Pierre Richelet, Dictionnaire français, 1693, «Faute » : «Balzac qui était un Grammairien a fait des fautes contre la Grammaire. Les Poëtes sont sujets à faire des grandes fautes de jugement ». On trouve, à " fausseté " : "Chose fausse. [C'est une fausseté indigne]. "

9 Antoine Furetière, Dictionnaire universel, I690, «Erreur » : « [...] La vanité humaine se repait de l'agréable erreur, de vivre bien loin dans l'avenir. Des H. Pline dit que c'est une heureuse erreur, que de croire ses amis plus parfaits qu'ils ne sont. Oev. M. ». 
La Manière de bien penser dans les ouvrages d'esprit révèle ainsi la singularité de la critique classique dans le rapport privilégié qu'elle entretient à l'erreur, qu'il s'agit de dénoncer et de corriger - que ce soit dans les " pensées » des poètes ou dans le goût du lecteur. La radicalité de cette optique nouvelle se justifie par la finalité morale dont elle se réclame. Ce sont ces deux aspects que l'on se propose d'examiner, non sans avoir au préalable inscrit l'œuvre du Père Bouhours dans la tradition rhétorique dont il se revendique tout en s'en distinguant.

\section{Le livre des erreurs}

Pour divertissante qu'elle soit, la forme dialoguée ne suffit pas à masquer l'érudition de l'auteur. Ces larcins, nombreux, sont d'ailleurs assumés en marge de l'ouvrage, où l'on retrouve les références convenues de l'éloquence classique : Quintilien, Cicéron, Horace. La construction même de l'ouvrage repose sur des notions héritées de cette tradition rhétorique. Ainsi, les trois genres définis par Eudoxe dans le second dialogue reprennent la tripartition cicéronienne entre style «simple », " moyen » et "élevé »". On reconnaît également, dans le dernier dialogue, les trois figures susceptibles d'induire en erreur selon Hermogène : l'antiphrase, l'équivoque et l'allusion ${ }^{11}$. La véritable originalité de l'ouvrage vient de l'ordre dans lequel ces matières sont traitées. La question de la "clarté " de la pensée n'est en effet abordée qu'en dernière partie, quand elle est le souci premier de la plupart des théoriciens ${ }^{12}$. Qui plus est, la synthèse opérée par Eudoxe en conclusion montre qu'au cours de leurs entretiens, les deux amis se sont moins préoccupés de définir «la manière de bien penser » que d'envisager toutes les manières qu'un auteur peut avoir de penser mal. Il apparait quaux quatre dialogues correspondent en effet les quatre erreurs possibles en matière de poétique : la fausseté, écart par rapport au «fonds de vérité " nécessaire à toute

10 Orator ad Brutum, respectivement $\$ 76-90,91-96$ et 97-99. Les trois genres distingués par Eudoxe sont le "noble ", "l'agréable » et le " délicat ", dont on peut penser qu'ils s'adaptent mieux aux catégories esthétiques de l'époque, en même temps qu'au sujet, "les ouvrages d'esprit ", que le Père Bouhours définit en préface comme " les histoires, les poèmes, les pièces d'éloquence, comme les harangues, les panégyriques, les oraisons funèbres, enfin tout ce qui s'écrit avec soin, et où il faut une certaine justesse qui va plus encore aux choses qu'aux paroles."

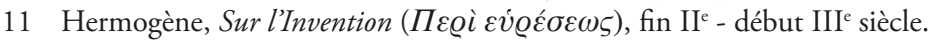

12 Dans sa contribution à l'ouvrage élaboré à son initiative et sous sa direction L'obscurité. Langage et herméneutique sous l'Ancien Régime, Delphine Denis revient sur la fortune de la "clarté " dans la tradition rhétorique. Elle rappelle ainsi que la "clarté " est citée par Aristote comme la "première qualité du style " (Rhétorique, III), qu'elle est le premier des « cinq flambeaux » de l'art oratoire selon Cicéron (Divisions de l'art oratoire, VI, 19), et la qualité première de l'élocution, dans la Rhétorique à Hérennius. (D. Denis, L’obscurité. Langage et herméneutique sous l'Ancien Régime, Louvain-la-Neuve, Academia-Bruylant, 2007). Même chez des auteurs contemporains de La Manière de bien penser, elle figure en tête des préoccupations : ainsi, Jacques Le Gras, dans sa Rhétorique française parue en 1671 (la même année que les Entretiens d'Ariste et d'Eugène du Père Bouhours) y consacre le premier chapitre du volume portant sur l'élocution. 
pensée ; l'inadéquation du style au sujet ; l'excès dans ce style (au "noble ", à " l'agréable » et au " délicat » se substituent alors "l'enflure », "l'afféterie », et le " raffinement ») et enfin "l'obscurité ».

Il en ressort une définition plus générale de l'erreur qui prend toujours la forme d'un " excès " par rapport à la " mesure " préconisée, d'un " écart " par rapport à un modèle clairement hérité des Anciens. La singularité de Bouhours vient de sa démarche de compilation. Dès le premier dialogue, il est précisé qu'Eudoxe se réfère à un " cahier " dans lequel sont rassemblées toutes les pensées défectueuses à ses yeux. La dynamique de l'entretien ne suffit d'ailleurs pas toujours à rompre la monotonie de cette énumération, et le recours à ce " cahier où [sont] ramassés divers exemples d'obscurité " ${ }^{13}$ est nécessaire pour rendre vraisemblable une telle accumulation. Ainsi, ce florilège est élaboré dans une perspective critique : à la faveur du dialogue, les deux amis vont relever les erreurs de certaines "pensées ", et parfois en proposer la correction. Cela passe notamment par la confrontation de pensées différentes, mais portant si possible sur un même objet : "Car pour vous faire mieux sentir le défaut d'une pensée qui est vicieuse en beau, il est bon de vous en dire quelques-unes en passant qui soient régulières, et correctes dans le même genre ${ }^{14}$. Afin de montrer en quoi Guarini ${ }^{15}$ pèche par excès, Eudoxe rapproche ainsi une de ses descriptions de celle de Virgile sur le même sujet :

Virgile dit que le Géant Encélade brûlé des foudres de Jupiter, vomit des flammes par les ouvertures de la montagne que les Dieux lui ont mise sur le corps ; et le Guarini dit que ce Géant lance des feux de colère et d'indignation contre le ciel, sans qu'on sache s'il est foudroyé, ou s'il foudroie. [...] L'un est naturel, et l'autre affecté. ${ }^{16}$

Selon cette perspective centrée sur le défaut plutôt que sur la perfection, les deux amis concluent par des recommandations aux auteurs. Pour commencer, il faudrait, dit Eudoxe, "qu'en écrivant ils eussent toujours devant les yeux diverses personnes comme témoins, et même comme juges de leurs pensées ${ }^{17}$. À rebours d'une formation rhétorique fondée sur l'imitatio, il ne propose donc pas des modèles, mais des censeurs. "Cela contenterait-il un tel ? " est la question que le poète doit garder à l'esprit. L'obsession de l'erreur doit être telle qu'on lui recommande au besoin d'aller jusqu'à formuler l'hypothèse d'un lecteur insuffisant : "Il ne serait pas même inutile au regard de la netteté et de la clarté, d'avoir en vue quelqu'un qui n'ait pas l'intelligence si pénétrante, ni la conception si aisée, et de se dire quelquefois, Monsieur tel

13 Dominique Bouhours, La Manière de bien penser..., op. cit., p. 365.

14 Ibid., p. 249.

15 Face à la référence classique de l'Énéide, l'auteur du Pastor fido incarne par excellence la pastorale italienne du dernier $\mathrm{XVI}^{\mathrm{e}}$ siècle.

16 Ibid., p. 231.

17 Ibid., p. 397. 
entendrait-il bien ma pensée ? » Et c'est Philanthe qui conclut : "Voilà sans doute de bons expédients [...] mais il m'en vient un qui serait infaillible à mon avis, et c'est de s'éloigner le plus qu'on peut du caractère de certaines gens que nous connaissons, et que j'ai admirées autrefois $[\ldots] »^{18}$. C'est donc par l'idée du contre-modèle que s'achève ce bref art poétique en négatif.

Le dialogue lui-même se suspecte d'erreur : "Vous et moi, avec toutes nos réflexions sur la fausseté des pensées, sommes capables de nous égarer, et nous nous égarons peut-être lors même que nous voulons redresser les autres ${ }^{19}$. Même scrupule au moment de conclure : "Mais en parlant de galimatias et d'obscurité, prenons garde d'y donner nous-mêmes : nous ne serions pas les premiers à qui cela serait arrivé ${ }^{20}$. Il faut ici rappeler que la perspective corrective est double : il ne s'agit pas seulement de former l'art du poète, mais aussi le goût du lecteur. Si le doux Philanthe est finalement à même de proposer des contre-modèles, c'est parce qu'il a lui-même été corrigé par l'entretien de son exigeant ami. On peut légitimement s'interroger sur les raisons qui autorisent à placer sur un même plan création et réception. Pour un lecteur de 1689, le rapport va de soi, et s'inscrit dans une démarche critique typique des années 1660, et que Delphine Denis qualifie de "nouvelle éthique de la lecture " :

Contre les « embarras " de l'expression qui opacifient la circulation du sens, le discours critique réunit sur un même front, dans le dernier tiers du XVII siècle, grammairiens, remarqueurs, gens du monde [...]. Après Valincourt puis l'abbé de Charnes " examinant " page à page le style de La Princesse de Clèves, on allèguera encore, en 1683, les Scrupules sur le style de Du Plaisir également attaché à débusquer la moindre équivoque ou amphibologie, au nom de l'" intérêt " du lecteur. ${ }^{21}$

Si le poète doit ainsi se garder scrupuleusement de tout écart ou ambiguïté, c'est parce qu'il a à charge un lecteur exposé à un même péril d'erreur. $L a$ Manière de bien penser est donc également le récit de formation de Philanthe, à qui Eudoxe enseigne à se garder des auteurs étourdis, sinon malveillants, qui entraînent le lecteur dans leur chute.

\section{Essai d'esthétique classique}

Apprenons avec Philanthe à nous garder de cette erreur de jugement qui ferait prendre une pensée pour bonne. L'une des confrontations les plus parlantes à cet égard est celle qu'opère Eudoxe entre deux épigrammes, l'un

18 Ibid., p. 398-399 : À la suite, Eudoxe mentionne tout de même l'inspiration par l'exemple, mais sans doute à des fins strictement encomiastiques, puisque cette remarque permet d'introduire immédiatement l'éloge d'un défunt orateur proposé comme un modèle.

19 Ibid., p. 74.

20 Ibid., p. 389.

21 Delphine Denis, L'obscurité. Langage et herméneutique sous l'Ancien Régime, art. cit., p. 28-29. 
de Voiture et l'autre de Saint-Amand. Le premier, loué pour sa justesse, traite de manière badine le sujet d'un accident du cardinal Mazarin, dont le carrosse avait versé :

Prélat passant tous les Prélats passés ;

Car les présents serait trop peu dire,

Pour Dieu rendez les péchés effacés

De ce Cocher qui vous sût mal conduire :

$S$ 'il fut peu caut à son chemin élire,

Votre renom le rendit téméraire.

Il ne crut pas versant pouvoir mal faire ;

Car chacun dit, que quoi que vous fassiez,

En guerre, en paix, en voyage, en affaire,

Vous vous trouvez toujours dessus vos pieds. ${ }^{22}$

À ce trait réussi, Eudoxe oppose le quatrain de Saint-Amand sur l'incendie du palais de Justice :

Certes l'on vit un triste jeu :

Quand à Paris Dame Justice

Se mit le Palais tout en feu

Pour avoir mangé trop d'épice. ${ }^{23}$

Si les deux amis s'accordent à trouver plaisant le mot de Voiture, ils ne s'entendent pas au sujet du second :

Ce quatrain a ébloui autrefois ; \& certaines gens le trouvent encore spirituel. Eh, qu'y a-t-il de plus heureux \& de plus joli, interrompit Philanthe ? Il ne se peut rien voir de plus creux ni de plus frivole, reprit Eudoxe; ce ne sont que des mots en l'air qui n'ont point de sens ; c'est du faux tout pur : car enfin, ce qu'on appelle épice au Palais n'a nul rapport à l'embrasement, \& le palais de la bouche qu'on a tout en feu, pour avoir mangé trop de poivre, ne conduit point à l'incendie d'un bâtiment où la justice s'exerce, $\&$ se vend si vous voulez. ${ }^{24}$

Pourquoi, selon Eudoxe, le mot de Voiture l'emporte sur celui de SaintAmand ? Quelle fut l'erreur de ce dernier ? Dans le premier cas, la situation concrète et l'expression abstraite, qui se rejoignent dans l'équivoque, ont pour référence Mazarin. L'accident de carrosse du "prélat ", s'il est un fait divers particulier, entre bien dans la catégorie générale des hasards de la fortune dont n'a cure le cardinal, et c'est bien ce talent qui reste souligné par le sens, littéral et figuré, de l'expression : "Vous retombez toujours dessus vos pieds". Les deux sens sont donc "vrais" au sens où l'entend Bouhours : l'équivoque est parfaitement exécutée. En revanche, la métaphore culinaire mobilisée par Saint-Amand " n'a nul rapport " à l'incendie du Palais de Justice. L'hyberbate d'Eudoxe (" et se vend si vous voulez ") met en lumière le principal reproche

22 Dominique Bouhours, La Manière de bien penser..., op. cit., p. 17-18.

23 Ibid., p. 20.

24 Ibid. 
qui est fait à Saint-Amand : tout au désir de faire la satire des juges, le poète fait un détour inutile par des jeux de sens entre des palais sans rapport, entre des épices sans relations (ceux qui relèvent un plat et ceux qui achètent les juges). C'est ce qui produit l'équivoque des "épices »: les revenus clandestins des juges ne provoquent pas l'incendie réel et ce feu demeure dans un sens figuré étranger au fait divers. De plus, en filant la métaphore gustative, les "épices " appellent une troisième équivoque sur le "palais ". Le reproche porte donc à la fois sur la forme et le fond, sur le style et sur la pensée : non seulement l'équivoque triple est d'une complexité qu'Eudoxe ne goûte pas, mais SaintAmand est coupable d'avoir inventé pour les besoins de sa satire un rapport de causalité qui n'a pas lieu d'être en vérité. Tout au long de son dialogue avec Philanthe, Eudoxe s'acharnera à relever les impossibilia, les paradoxes, les excès et les confusions. Il leur oppose systématiquement le souci de la référence, et un idéal de la "pensée » comme objet clos, resserré et auto-référent. On reconnait avec Jean Rousset la critique du classique contre le baroque :

La beauté classique, Eudoxe la compare à un vêtement bien ajusté, qui fait paraître la taille et se modèle sur le corps. Ni nudité, ni voiles flottant amplement autour du corps. Quand le goût classique prend conscience de lui-même, comme il le fait ici avec beaucoup de netteté et de délicatesse, c'est donc contre le Baroque, contre les défauts baroques, perçus comme des erreurs étrangères au goût français, cultivées de préférence hors de France. ${ }^{25}$

Il convenait donc que le galant Voiture reçût des éloges, tandis que SaintAmand le burlesque était blâmé.

Toutefois, les critiques d'Eudoxe frappent par leur insistance à faire preuve d'objectivité. Dès le début de l'œuvre, à Philanthe qui lui reproche de calquer son goût sur celui d'un certain Provincial dont il lit les Doutes sur la langue française, il répond : "Ce n'est pas sur le Provincial que je me suis réglé [...] c'est sur le bon sens qu'il prend lui-même pour sa règle dans ce qui ne dépend pas précisément de l'usage ${ }^{26}$. Plus loin, il affirme qu' « il ne faut que consulter la raison pour n'approuver pas certaines pensées que tout le monde presque admire » et que " rien n'est moins raisonnable que d'avoir des pensées sublimes dans un petit sujet qui n'en demande que de médiocres ${ }^{27}$. On ne peut que souligner les accents cartésiens du personnage. De plus, à la place de la clarté - notion ordinairement prépondérante dans l'esthétique classique, et dont on a vu qu'elle était ici reléguée au dernier entretien - Eudoxe préfère mettre en avant l'exigence de "vérité ":

25 Jean Rousset, La Littérature de l'Âge baroque en France, op. cit., p. 245.

26 Manière de bien penser..., p. 4. Les soupçons de Philanthe sont justifiés : la mention des Doutes sur la langue française proposés à Messieurs de l'Académie par un gentilhomme de Province signale Eudoxe comme l'avatar du Père Bouhours, qui en est l'auteur...

27 Ibid., p. 80. 
Car enfin pour vous dire un peu par ordre ce que je pense là-dessus ; la vérité est la première qualité, \& comme le fondement des pensées : les plus belles sont vicieuses ; ou plutôt celles qui passent pour belles, \& qui semblent l'être, ne le sont pas en effet, si ce fonds leur manque. ${ }^{28}$

On pourrait arguer que cet ordre se justifie par la dimension propédeutique de l'œuvre, qui veut que Philanthe aborde en premier lieu le «fonds » des pensées pour finir par leur forme.

Il n'en est pas moins remarquable que ce choix ressortit à la démarche analytique initiée par l'auteur du Discours de la méthode ${ }^{29}$. Il n'est pas question ici de faire des rapprochements entre Descartes et le Père Bouhours, ni même de chercher si, oui ou non, le père jésuite pouvait être appelé un cartésien. Il s'agit seulement de souligner que l'auteur de La Manière de bien penser personnifie en Eudoxe ce que Michel Foucault désigne par l'épistémè clas$s_{i q u e} e^{30}$ dans ce qu'elle a surtout de discriminant. Plusieurs passages illustrent cette volonté de s'affranchir d'un classement des auteurs déjà regardé comme traditionnel, au profit d'une forme d'objectivité critique, qui doit en dernière instance permettre à tout poète de se garder des erreurs. C'est Philanthe qui commence par relever des fautes chez Voiture ${ }^{31}$, puis chez Malherbe ${ }^{32}$. Eudoxe reconnait les erreurs sans faire de difficultés : "Ce n'est pas par ces endroitslà $[. .$.$] que j'estime et que j'admire Malherbe : il y sort visiblement de son$ caractère, et je ne l'y reconnais pas. [...] Je suis de bonne foi, dit Eudoxe, et l'amitié ne m'aveugle pas jusqu'à ne point voir les défauts de mes amis ${ }^{33}$. Là où Philanthe investit de la " colère ", de la "passion " ${ }^{34}$, Eudoxe fait preuve de maîtrise de soi et de distance critique.

Cette « raison » nouvellement affirmée entre malgré tout en conflit avec une autre forme de critique, fondée sur le jugement de "goût ". Le terme est employé plusieurs fois, de préférence par Philanthe, qui valorise le plaisir du texte : il est

28 Ibid., p. 12.

29 Paru en 1637 chez Jan Maire comme préface à la Dioptrique, aux Météores et à la Géométrie, ce discours sera, comme on le sait, une source d'inspiration dans de nombreux domaines du savoir, et pour les philologues en particulier. La Grammaire de Port-Royal se revendique d'ailleurs de la méthode cartésienne (Grammaire générale et raisonnée contenant les fondements de l'art de parler, expliqués d'une manière claire et naturelle, P. Le Petit, Paris, 1660).

30 Michel Foucault, Les mots et les choses. Une archéologie du savoir en sciences humaines, Paris, Gallimard, 1966.

31 Dominique Bouhours, La Manière de bien penser..., op. cit., p. 262 : " Mais savez-vous bien, repartit Philanthe un peu en colère, que votre Voiture est quelquefois ampoulé lui-même, et que la première lettre a beaucoup de ce sublime qui ne vous plait pas?"

32 On peut lire à propos de La Pénitence de Saint Pierre : "Malherbe du moins, répliqua Philanthe, qui vous semble et si sensé et si juste, ne l'est pas toujours. Il est ampoulé en de certains rencontres [...] ». (Ibid., p. 267)

33 Ibid., respectivement p. 267 et 295.

34 Op. cit., p. 336 : «Quelque passion que j’aie toujours eue pour Balzac, dit Philanthe, je ne puis nier que cela ne soit un peu quintessencié. " 
notamment le sujet du premier exemple qui lui vient à l'esprit pour illustrer un certain type d'obscurité ${ }^{35}$. Eudoxe souligne ailleurs les contradictions qui ne manquent jamais de survenir à son sujet, par exemple chez La Rochefoucauld, qui affirme à la fois "Le bon goût vient plus du jugement que de l'esprit » et "Quand notre mérite baisse, notre goût baisse aussi » ${ }^{36}$. Et d'ironiser :

[...] Il y a là une délicatesse qui me passe, et c'est peut-être ma faute. Il me semble, dit Philanthe, que j'ai entendu cette réflexion toutes les fois que je l'ai lue; car j'ai lu plus d'une fois les Réflexions morales: mais je ne l'entends pas plus que vous présentement, et je crois que nous avons tous deux l'esprit bouché. ${ }^{37}$

Contrairement à la "vérité » ou à la " clarté », le goût n’est pas défini dans le cours de l'entretien, mais seulement indirectement à travers des citations dont on examine surtout le style. Dans ce dernier dialogue, Philanthe reconnait à demi-mot que cette notion qu'il a "entendue " jusque-là ne lui semble plus si claire, non seulement telle qu'elle est définie par La Rochefoucauld, mais dans l'absolu : il n'est pas interdit de lire, derrière l'ingénuité de son désarroi, une certaine ironie de l'auteur. Nous ne sommes plus qu'à quelques pages de sa reddition : "Comme je suis de bonne foi, repartit Philanthe, je vous avoue franchement, mon cher Eudoxe, que je vois maintenant les choses avec d'autres yeux, et que mon goût n'est presque plus différent du vôtre ${ }^{38}$. C'est faute d'avoir levé l'ambiguïté de la notion. En effet, comment expliquer que l'épigramme de Saint-Amand, dont Eudoxe a démontré l'erreur selon les critères de la "raison ", ait pu faire rire Philanthe ? Curieusement, la défense favorite des dramaturges de l'époque, "le goût du public ", la spontanéité du rire ou des larmes suscités par l'œuvre, n’est jamais avancé. Cela ne va pas sans quelques contradictions : au moment même où Philanthe s'exclame : "Ah que cela est joli ! », Eudoxe reprend : " Prenez garde [...] que les métaphores tirées de ce que la nature a de plus doux et de plus riant, ne plaisent guères que quand elles ne sont point forcées! " ${ }^{39}$ Faut-il alors déduire de cette nouvelle règle curieusement exprimée que ces métaphores qui font les délices

35 Il s'agit de la confusion suscitée par le chevauchement de deux réseaux métaphoriques (ici encore, un procédé caractéristique du baroque) : «Plusieurs métaphores entassées les unes sur les autres font aussi ce mauvais effet [...] Il arrive même que deux métaphores qui ne sont pas dans le même genre, étant jointes, diminuent quelque chose de la clarté d'une pensée. Je vous comprends, dit Philanthe, et je vois maintenant pourquoi la pensée d'une personne savante bien au-dessus de son sexe, qui a entrepris de nous expliquer ce que c'est que le goût en matière d'esprit, et qui l'a fait d'une manière si délicate ; pourquoi, dis-je, sa pensée, qui est au fonds vraie et solide, ne m’a pas paru d'abord extrêmement claire ; c'est sans doute qu'elle définit le goût, qui est une métaphore, par l'harmonie qui en est une autre d'un genre différent. Car, si je m’en souviens, voici la définition : Le goût est une harmonie, un accord de l'esprit et de la raison. " (Ibid., p. 380)

36 La Rochefoucauld, Réflexions ou sentences et maximes morales, 258, éd. L. Plazenet, Champion, Paris, 2002, p. 166.

37 Dominique Bouhours, La Manière de bien penser..., op. cit., p. 381.

38 Ibid., p. 391.

39 Ibid., p. 292. 
de Philanthe "ne sont point forcées "? Non, et bien au contraire. Contre la loi du goût, qui veut que ce qui plait à certains a nécessairement quelque chose d'aimable, cette seconde loi soulève une difficulté : d'après elle en effet, Philanthe ne devrait pas pouvoir trouver agréable cette pensée, qui commet l'erreur d'être outrée. Cette situation se reproduira plusieurs fois au cours du dialogue, et ne laissera pas de mettre la raison d'Eudoxe en difficulté : "La pensée me semble belle et délicate, dit Philanthe. Elle a, repartit Eudoxe, un peu plus de délicatesse qu'il ne faut, et si vous ne vous en apercevez pas, je ne sais comment vous le faire entendre : on sent cela mieux qu'on ne l'explique ${ }^{40}$. Ces hésitations n'empêcheront pas qu'à la fin Philanthe ne se défasse de ses " anciennes erreurs " ${ }^{41}$ : ce sont les mots d'Eudoxe, et la conclusion d'un débat qui désormais n'a plus lieu d'être.

\section{Philanthe "guéri de son erreur» : une éthique poétique}

La dimension morale de l'erreur telle qu'on l'a vu soulignée par les dictionnaires de l'époque se retrouve dans la manière dont Eudoxe parle des mauvaises pensées tout au long de l'ouvrage. Qualifiées de "fautes », elles sont "vicieuses », elles " pèchent contre » le bon sens. À ce lexique se joignent parfois des métaphores qui reprennent tous les traits que l'on a coutume de voir épinglés par les moralistes. L'obscurité, par exemple, est totalement discréditée :

À parler en général, poursuivit Eudoxe, tout Écrivain, soit Historien ou Philosophe, soit Orateur ou Poète, ne mérite pas d'être lu, dès qu'il fait un mystère de sa pensée. C'est comme ces femmes qui vont masquées par les rues, ou qui se cachent dans leurs coiffes, et qui ne veulent pas qu'on les connaisse : il faut les laisser passer, et ne les regarder pas seulement. ${ }^{42}$

Bouhours va parfois jusqu'à construire un caractère : c'est notamment le cas lorsqu'il aborde la question de la langue ${ }^{43}$. Si les deux amis conviennent

40 Ibid., p. 310.

41 Ibid., p. 393.

42 Ibid., p. 369.

43 Le topos de la langue française comme langue universelle de la pensée, excellant par la clarté qu'elle devrait à sa syntaxe, connait alors une grande fortune : l'auteur l'avait exploité dans l'un des Entretiens d'Ariste et d'Eugène, en développant une apologie inconditionnelle du français, au détriment assumé des autres langues. Celles-ci étaient en effet considérées dans les Entretiens comme véritablement impropres à l'expression d'une pensée vraie. En effet, défendant une critique fondée sur le critère de la mimesis, l'auteur développait une analogie entre parler et peindre, affirmant que " chaque langue est un art particulier de rendre ces conceptions de notre esprit sensible, de les faire voir ". À ce titre, l'Espagnol et l'Italien étaient disqualifiés au motif que le premier "va plus loin que la nature », tandis que le second « songe plus à faire de belles peintures que de bons portraits ». Pour l'auteur, il n'y a que la langue française "qui sache bien peindre d'après nature ", de sorte que toute pensée exprimée autrement qu'en français est suspecte d'une erreur inhérente à sa langue : il serait impossible de dire quelque chose de vrai en italien, en espagnol ou en allemand. (Dominique Bouhours, Entretiens d'Ariste et d'Eugène, éd. B. Beugnot et G. Declerq, Paris, Champion, 2003, p. 62-65). 
dans le premier dialogue que toutes les langues sont tenues à la même exigence de vérité ("Comme si la justesse de sens [...] n’était pas de toutes les langues, et que ce qui est mauvais de soi-même dût passer pour bon en aucun pays parmi les personnes raisonnables $\left.{ }^{44}\right)$, le dernier entretien viendra nuancer cette affirmation. Eudoxe y explique en effet que l'italien et l'espagnol ont non seulement une tendance naturelle à l'erreur, mais à une certaine erreur qui leur est propre. Après avoir opposé Le Tasse à Virgile, il conclut ainsi :

Les Poètes Italiens ne sont guères naturels, il fardent tout [...] Le Tasse, qui est un si beau génie, tient un peu du caractère des femmes coquettes, qui mettent du fard, quelques belles qu'elles soient, sans prendre garde que l'artifice gâte en elles la nature, et qu'elles plairaient davantage si elles avaient moins envie de plaire. ${ }^{45}$

Ce portrait de la langue italienne, à travers Le Tasse, n'est pas sans rappeler l'Iphis de La Bruyère, qui "n'oublie pas de s'embellir "46. Le Père Bouhours représente ici un véritable caractère, ce qui lui permet de faire basculer sa critique du domaine de l'esthétique à celui de la morale.

Les conséquences éthiques de l'erreur poétique ne sont cependant pas uniquement métaphoriques. Le cas de la flatterie dans les genres encomiastiques est ainsi très concret, et les reproches sont virulents à l'encontre des poètes qui s'y livrent. Les excès de Martial à l'égard de Domitien sont ainsi plus d'une fois condamnés. Le poète s'est rendu coupable d'impiété dans cet épigramme : "Différez, je vous prie, César, le plus que vous pourrez d'aller prendre place à la table de Jupiter ; ou venez ici vous-même, Jupiter, si vous êtes pressé d'avoir un tel convive que César ${ }^{47}$. Le contexte païen n'atténue pas la gravité de l'offense faite au "Maître des Dieux " $^{48}$. Philanthe croit pouvoir, ici encore, le justifier par le genre dans lequel ces éloges s'inscrivent, mais le jugement d'Eudoxe est sans appel : «C'est une flatterie, dit Philanthe. Je l'avoue, repartit Eudoxe ; mais c'est une flatterie qui blesse la Religion et le bon sens tout ensemble ${ }^{49}$. Les deux amis s'accordent finalement sur la bassesse de ces fausses louanges : "C'est la flatterie, dit Philanthe, qui a introduit ces pensées. Oui, reprit Eudoxe : à mesure que la liberté diminua parmi les Romains, et que les tyrans devinrent plus maitres, la générosité et le bon sens s'altèrent; la flatterie devint plus lâche et mieux raisonnable " ${ }^{50}$. La critique morale se revendique ainsi de deux éthiques : religieuse et politique.

44 Dominique Bouhours, La Manière de bien penser...., op. cit., p. 41.

45 Ibid., p. 236-237.

46 Jean de La Bruyère, Les Caractères, 6e édition, 1691, « De la Mode ».

47 Martial, Épigrammes, VIII, Ie siècle ap. J.-C.

48 Dominique Bouhours, La Manière de bien penser..., op. cit., p. 255.

49 Ibid.

50 Ibid., p. 259-260. 
La mise en scène des entretiens vient parachever cette perspective morale, puisque le dialogue est enchâssé dans un récit analogique du discours. Ainsi, au moment où Eudoxe s'apprête à aborder les excès du style, il est interrompu par la visite de quelques "fâcheux " : c'est cette intervention qui marque la transition vers la troisième partie, ayant pour sujet ces poètes qui excèdent la mesure requise, contrastant avec la retraite finalement préférée par les deux amis : « un certain endroit écarté où règne un profond silence, et qui a tous les charmes de la solitude ${ }^{51}$. De même, dans le dernier dialogue qui traite du danger de l'obscurité, les deux amis doivent garder la chambre car le temps s'est assombri. Mais surtout, la correction du goût de Philanthe a des répercussions morales. L'auteur nous dit ainsi que le lendemain du premier dialogue, "Il se leva de bonne heure contre sa coutume, et alla aussitôt chercher Eudoxe que l'amour de l'étude rend fort matineux, à l'exemple de ces Philosophes [... $»^{52}$. Au fur et à mesure que son ami lui fait voir ses erreurs, tous deux se rangent à un idéal de retraite et d'étude austère. Discrètement toutefois, les corrections successives opérées par ce sévère ami ont pour effet de soustraire petit à petit, avec l'erreur, un autre aspect essentiel de la lecture : le plaisir. On a déjà vu que Philanthe pouvait, à la faveur de l'entretien, ne plus comprendre certaines pensées qui lui avaient paru limpides : il arrive finalement qu'elles ne le touchent plus : "Je vous avoue néanmoins [...] que ce poignard qui ne rougit que du sang de Pauline, comme s'il avait honte d'avoir blessé une femme, me plait un peu moins aujourd'hui qu'il ne me plaisait autrefois ${ }^{53}$. Eudoxe est ainsi amené à disséquer et critiquer jusqu'à l'anéantissement les œuvres favorites de son ami, qui essaie désespérément d'en sauver certains traits, souvent sans succès : "En vérité, repartit Philanthe, vous renversez toutes mes idées. Croyez-moi, reprit Eudoxe, il ne faut jamais s'égayer trop, même dans les matières fleuries ; et il vaudrait presque mieux qu'une pensée fut un peu sombre, que d'être si brillante ${ }^{54}$. Revenir de l'erreur ne se fait pas sans une certaine mélancolie, qui éclate particulièrement dans le discours d'Eudoxe au moment où Philanthe achève de rendre les armes :

Je sens, ajouta-t-il, que la lecture des Italiens et des Espagnols ne me plaira pas tant qu'elle faisait. Vous serez, interrompit Eudoxe, comme ces gens qui sont détrompés du monde, et qui dans le commerce de la vie n'ont pas tant de plaisir que les autres : mais assurez-vous que c'en est un grand d'être détrompé, et ne vous avisez pas d'imiter ce fou, qui s'imaginait être toujours au Théâtre, et entendre d'excellents Comédiens ; mais qui étant guéri de son erreur par un breuvage que ses amis lui firent prendre, se plaignait de ses amis comme s'il l'eussent assassiné. ${ }^{55}$

51 Ibid., p. 242.

52 Ibid., p. 77.

53 Ibid., p. 340.

54 Ibid., p. 283.

55 Ibid., p. 391-392. 
L'entretien s'achève sur l'image d'un critique exclu du monde, et la dernière recommandation d'Eudoxe est prétexte à l'éloge funèbre d'un proche del'auteur ${ }^{56}$. Par son évolution progressive, le dialogue introduit un paramètre essentiel dans la perspective corrective de l'œuvre, qui est l'effet du temps. Eudoxe reconnait ainsi n'avoir pas toujours rejeté ce que Philanthe goûte aujourd'hui :

Les premières pensées qui me viennent là-dessus sont de la Métamorphose des yeux de Philis changés en Astres ; vous connaissez ce petit ouvrage. C'est un chefd'œuvre d'esprit, dit Philanthe, et j'en suis charmé toutes les fois que je le lis. J'en ai été charmé comme vous, reprit Eudoxe ; mais j'en suis bien revenu, et je n'y admire plus guère que l'affectation. ${ }^{57}$

Plus qu'ailleurs, on peut reconnaître ici les propres mots de Bouhours face à la rupture esthétique de 1665, qui fait écrire à Jean Rousset : «Bouhours ou Chevreau parlent de leur jeunesse comme d'un autre temps, où le goût n'était pas le même, où l'on aimait des œuvres dont ils se disent depuis longtemps revenus ${ }^{58}$. La nouveauté vient de ce que cet ancien goût n'est pas distingué comme seulement passé de mode, mais comme une erreur qu'il faudrait corriger - quoiqu'on n'en revienne qu'avec nostalgie.

Par sa forme hybride du florilège, de l'entretien, de l'analyse et du récit, $L a$ Manière de bien penser est un miroir fidèle des préoccupations poétiques d'une époque de transition. Le dialogue d'Eudoxe et Philanthe est bien celui du classicisme avec le baroque. Cependant, au "menuet pédagogique " $"$ qui équilibrait les Entretiens d'Ariste et d'Eugène seize ans auparavant, succède le ton didactique d'un personnage favorisé par l'auteur, et parvenant finalement à corriger son cadet. Cette évolution est aussi celle de l'humanisme, qui compile et compare, vers la modernité : un classicisme non seulement esthétique, mais épistémique, qui discrimine et critique.

Rien d'étonnant dès lors à ce que la voie opposée aux erreurs se caractérise, en poétique comme en morale, par la mesure. Eudoxe cite Scaliger pour dénoncer l'erreur qui consiste à chercher "au-dessus de ses forces $»^{60}$, mais on aurait pu emprunter le même conseil à La Fontaine : " ne forçons point notre talent $~_{61}$. La Manière de bien penser soulève en poétique la même difficulté que les moralistes dans leur domaine : celle du conflit entre la singu-

56 Ibid., p. 399. Il est précisé en marge qu’il s’agit de « M. Pageau, célèbre avocat. "

57 Ibid., p. 282.

58 Jean Rousset, La Littérature de l'Âge baroque en France, op. cit., p. 235.

59 Bernard Beugnot, dans sa préface à l'édition critique des Entretiens d'Ariste et d'Eugène, emprunte l'expression à la thèse d'Ugo Dionné, Dialogue et digression au XVII' siècle : les Entretiens sur les vies des peintres d'André Félibien, Montréal, 1995, sous la direction de Françoise Siguret.

60 Dominique Bouhours, La Manière de bien penser..., op. cit., p. 242 : "Conatus supra vires et supra rem., J.C. Scaliger, Poetices libri VII ».

61 Jean de La Fontaine, Fables, IV, 5, «L'Âne et le Petit Chien », Paris, D. Thierry, 1668. 
larité contingente d'un goût - un caractère - et l'universel impératif de la règle. La conclusion du Père Bouhours laisse toutefois entendre qu'en cette fin de siècle, l'équilibre entre les deux soit en passe d'être brisé, au profit des rigueurs de la seconde. La création poétique, comme la lecture, sont finalement perçues comme des voies d'accès à la vérité : dans la variété des chemins qui s'y rencontrent, il faut en préférer un, et se détourner des autres comme d'autant d'" erreurs".

Si le terme même n'apparait qu'à la fin des entretiens, c'est parce que la notion est le fruit d'une élaboration tout au long de l'ouvrage. Tant que dominent les critères du goût et du plaisir, elle ne peut en effet avoir cours. Mais dès que Philanthe accepte de se ranger à un " goût " unique, cette idée fait place à une alternative à deux termes, et le jeune homme s'en retourne à la ville "fort satisfait de sa visite, et bien résolu de se déclarer partout pour le bon sens contre le faux bel esprit. " ${ }^{62}$ L'intérêt de la Manière de bien penser vient moins, pour le lecteur contemporain, de l'affirmation de la supériorité d'une esthétique sur une autre, que de la conception critique dont elle témoigne : la création poétique, désormais envisagée comme une entreprise éthique à l'égard du lecteur, consiste pour l'auteur à choisir entre de bonnes et de mauvaises pensées, en se référant si besoin au discours normatif qui doit être celui du critique moderne. Cette évolution ne va pas sans une certaine nostalgie : dans ce dernier mouvement, le Père Bouhours semble tristement partagé entre l'attitude moderne d'une époque dont la mort récente d'un ami lui rappelle qu'elle n'est pas la sienne, et le souvenir d'une jeunesse enthousiaste aux goûts passés de mode. S'il reconnait cette nouvelle conception poétique, il ne peut se résoudre à y souscrire tout-à-fait : tout en rejetant ce qu'il convient désormais de qualifier d' " erreurs ", il continue de les compiler, geste caractéristique d'un esprit humaniste qui ne peut se résoudre à la suppression, dans un espoir de perfectibilité plutôt que dans un idéal de perfection. L'idéal du père jésuite s'illustre dans son ouvrage même : un discours dont la vérité serait garantie par un garde-fou de citations marginales héritées des Anciens, et dont l'immédiate présence aux côtés du dialogue le plus contemporain rendrait sensible la permanence de la pensée vraie.

Raphaëlle Longuet

UMR 8599 CELLF

Université Paris-Sorbonne rlonguet@ac-versailles.fr 


\section{Résumé}

Dans un entretien didactique visant à former le goût du lecteur à travers un florilège critique des erreurs du discours, le Père Bouhours affirme que la vérité doit constituer la qualité première de la pensée : elle devient ainsi paradoxalement le souci principal de tout jugement de goût, et conduit l'auteur à bannir du discours toutes les figures qui, loin de le perfectionner, le dénaturent.

\section{Mots-clefs}

Erreur, goût, jugement, Bouhours, pensée.

\section{Abstract}

In a didactic conversation between two characters, Bouhours, basing himself on mistakes taken from famous works, considers the truth as paramount in "pensées" writing and reading, which paradoxically becomes a criterion for the judgment of taste, and bounds compels the writers to avoid rhetorical figurestropes, which therefore appear to be less of a perfecting of speech, than a denaturation of the speech thereof.

\section{Keywords}

Mistake, taste, judgment, Bouhours, pensée. 